\title{
Thymidine-dependent Escherichia coli infection and some associated laboratory problems
}

\author{
ELIZABETH I. TANNER AND C. H. BULLIN \\ From the Public Health Laboratory, West Park Hospital, Epsom, Surrey
}

SYNOPSIS The rapid emergence of a thymidine-dependent strain of Escherichia coli in a patient treated with co-trimoxazole is described. The organism was recovered from blood cultures during a septicaemia which developed after only a few days of co-trimoxazole treatment. The cultural characteristics of three thymidine-dependent strains were studied and a remarkable variation was found in their ability to grow on different batches of blood agar, depending upon the age of the plates and the type of agar base used.

There have been several reports of thymidinedependent enterobacterial infection in patients treated with co-trimoxazole (Barker, Healing, and Hutchinson, 1972; Okubadejo and Maskell, 1973) and in most of these cases the organisms have been grown from specimens of urine. We have recently isolated thymidine-dependent strains of Escherichia coli from the blood of a patient with septicaemia and from the urine of two other patients; all three had received co-trimoxazole therapy. This report describes these cases and the laboratory problems involved in their diagnosis and treatment.

\section{Clinical Summaries}

CASE 1

A woman (D.F.), aged 62 years, was admitted to hospital complaining of pain in the right loin, nausea, and vomiting for several hours. She had previously been healthy apart from several attacks of cystitis in recent years. On examination she was apyrexial and tender in the right loin.

During the next 48 hours her temperature rose to $39 \cdot 5^{\circ} \mathrm{C}$ and she had a rigor. A diagnosis of acute pyelonephritis was made. A blood culture was taken and co-trimoxazole treatment commenced at a dosage of 2 tablets twice daily (one tablet contains $80 \mathrm{mg}$ trimethoprim and $400 \mathrm{mg}$ sulphamethoxazole). Ward testing revealed haematuria and proteinuria but urine was not cultured before treatment was started.

The blood culture yielded an $E$. coli sensitive to trimethoprim (minimum inhibitory concentration $0.5 \mu \mathrm{g} / \mathrm{ml}$ ) and ampicillin (MIC $4.0 \mu \mathrm{g} / \mathrm{ml}$ ) but

Received for publication 27 March 1974. resistant to sulphonamide ( $\mathrm{MIC}>2000 \mu \mathrm{g} / \mathrm{ml}$ ). The temperature initially subsided (see fig)and the patient felt better but after a few days the pyrexia returned. Co-trimoxazole was discontinued and after 24 hours further blood cultures were taken. Five sets of blood cultures collected during the ensuing 24 hours yielded a thymidine-dependent strain of $E$. coli in 12 of the 15 bottles. Intravenous ampicillin was given in a dosage of $1 \mathrm{~g}$, four hourly, and the temperature returned to normal within a few days. Urine samples were taken at intervals (see fig) but at no time was any significant bacterial growth obtained. An intravenous pyelogram revealed no abnormality.

A month after discharge from hospital the patient complained of nocturia; her urine contained 25 cells per $\mathrm{cmm}$ and yielded a significant growth of a nonthymidine-dependent strain of $E$. coli, sensitive to nitrofurantoin and trimethoprim but resistant to ampicillin and sulphonamide. She was given a course of nitrofurantoin and when next seen her urine was clear.

CASE 2

A man (T.T.), aged 26 years, had an indwelling catheter following a partial cystectomy. E. coli sensitive to trimethoprim, resistant to sulphonamide, ampicillin, and tetracycline, was isolated from the urine. He was given a 10-day course of cotrimoxazole and six days later a thymidine-dependent strain of $E$. coli resistant to ampicillin, tetracycline, and sulphonamide was isolated from his urine and abdominal wound.

CASE 3

A woman (E.H.), aged 70 years, suffering from 


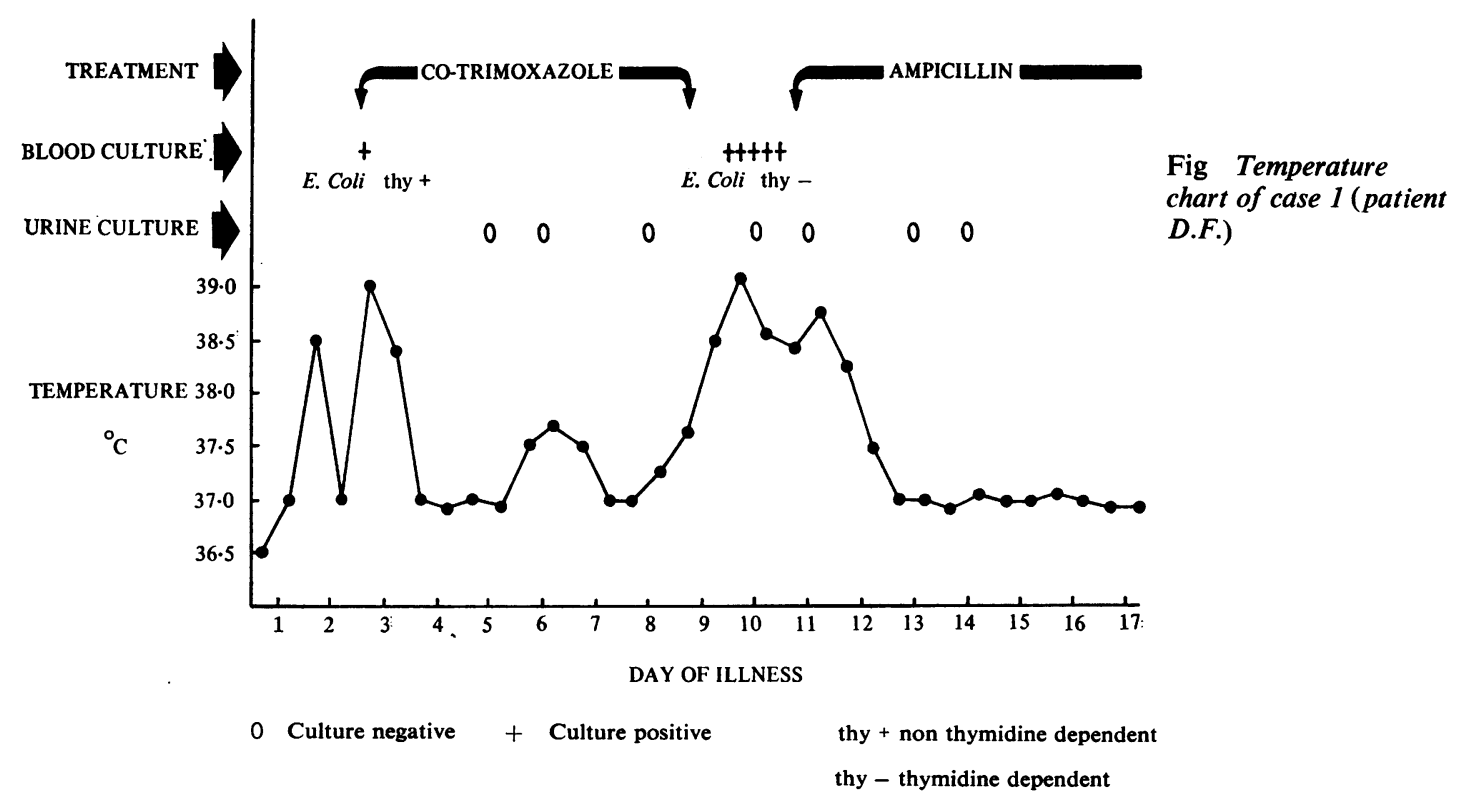

multiple sclerosis, had a permanent indwelling catheter. Following a course of co-trimoxazole she was given ampicillin and during the latter treatment a thymidine-dependent strain of $E$. coli resistant to ampicillin was isolated from her urine.

\section{Laboratory Investigations}

\section{MATERIALS AND METHODS}

The media used were Oxoid blood agar base no. 2 containing $7 \%$ horse blood, Oxoid MacConkey agar no. 2, Oxoid CLED medium (cysteine, lactose, electrolyte-deficient agar), and a lysed blood agar (Oxoid diagnostic sensitivity test agar containing $7 \%$ lysed horse blood). Oxoid Columbia agar base was included for comparative tests of thymidine content. For blood cultures Oxoid nutrient broth no. 2 containing $0.05 \%$ sodium polyanethol sulphonate was used.

Antibiotic sensitivity tests employed discs containing ampicillin $10 \mu \mathrm{g}$, sulphonamide $100 \mu \mathrm{g}$, trimethoprim 1.25 $\mu \mathrm{g}$, nitrofurantoin $200 \mu \mathrm{g}$, and tetracycline $10 \mu \mathrm{g}$. Inhibition zone diameters were compared with those given by the control $E$. coli NCTC 10418. The MIC of ampicillin was estimated by a broth dilution method, and of sulphonamide and trimethoprim by a plate dilution method in lysed blood agar. For both methods the inoculum was $0.2 \mathrm{ml}$ of a $10^{-2}$ dilution of an overnight broth culture and the control $E$. coli was tested in parallel.

The organisms were identified as $E$. coli by conventional techniques (Cowan and Steel, 1966). Their nutritional requirements were tested on minimal agar (Difco Davis minimal broth with $1.5 \%$ Oxoid agar no. 1 and $0.1 \%$ glucose) to which were added varying concentrations of thymine or thymidine. Twelve colonies of each mutant strain and the control $E$. coli were scraped from CLED plates, washed three times in minimal broth, and resuspended in minimal broth to give a faintly turbid suspension. One standard loopful was spread to give individual colonies and plates were assessed after 48 hours' incubation. The amount of growth and size of colonies were compared with the growth of the same organism on CLED medium.

\section{Results}

The initial blood culture from case 1 yielded an $E$. coli which grew normally on the usual media. Following the relapse blood cultures yielded an $E$. coli with altered cultural characteristics. After 24 hours' incubation, aerobic and anaerobic subcultures on blood agar produced a variable scanty growth of minute colonies $(0.5-1 \mathrm{~mm}$ diameter) of a pleomorphic Gram-negative rod. This organism was further subcultured and it was found to grow well on nutrient, chocolate, CLED, and MacConkey agars, resembling typical $E$. coli colonies. It grew poorly, however, on blood agar, and not at all on lysed blood agar, unless a thymidine-impregnated strip was placed on the agar when normal growth occurred around the strip. Discs, impregnated with sulphonamide, nitrofurantoin, p-aminobenzoic acid, or 


\begin{tabular}{|c|c|c|c|c|c|c|c|c|c|c|c|}
\hline \multirow[t]{2}{*}{ Organism } & \multicolumn{5}{|c|}{ Thymidine $\left(\mu \mathrm{g} / m l^{1}\right)$} & \multicolumn{5}{|c|}{ Thymine $(\mu g / m l)$} & \multirow[t]{2}{*}{$C L E D$} \\
\hline & 1 & $0 \cdot 1$ & 0.01 & 0.001 & 0 & 1 & $1 \cdot 0$ & $0 \cdot 1$ & 0.01 & 0 & \\
\hline $\begin{array}{l}\text { Control } E \text {. coli } \\
\text { Thymidine-dependent D.F. } \\
\begin{array}{ll}\text { E. coli } \text { strain } & \text { T.T. } \\
& \text { E.H. }\end{array}\end{array}$ & $\begin{array}{l}++^{2} \\
++ \\
++ \\
++\end{array}$ & $\begin{array}{l}++ \\
+3 \\
++ \\
++\end{array}$ & $\begin{array}{l}++ \\
+ \\
+ \\
+\end{array}$ & $\begin{array}{l}++ \\
- \\
-\end{array}$ & $\begin{array}{l}++ \\
- \\
-\end{array}$ & $\begin{array}{l}++ \\
++ \\
++ \\
++\end{array}$ & $\begin{array}{l}++ \\
++ \\
- \\
++\end{array}$ & $\begin{array}{l}++ \\
- \\
+\end{array}$ & $\begin{array}{l}++ \\
- \\
-\end{array}$ & $\begin{array}{l}++ \\
- \\
-\end{array}$ & $\begin{array}{l}++ \\
++ \\
++ \\
++\end{array}$ \\
\hline
\end{tabular}

Table I Thymine and thymidine requirements of thymidine-dependent $\mathrm{E}$. coli mutants

${ }^{1}$ In minimal medium

${ }^{2}$ Growth similar to that on CLED

$+{ }^{3}$ Tiny colonies

- No growth

cysteine, failed to promote growth which was apparently dependent on the presence of thymidine.

Assays of the thymidine and thymine requirements of strains D.F., T.T., and E.H. (cases 1, 2, and 3, respectively) gave the results shown in table $I$. These strains all required $0.01 \mu \mathrm{g} / \mathrm{ml}$ of thymidine to produce visible colonies; the thymine requirement was more variable but on average $1 \mu \mathrm{g} / \mathrm{ml}$ was necessary-one hundred times the thymidine requirement. The effect of thymidine was abolished by the addition of $7 \%$ lysed horse blood, presumably as a result of the conversion of thymidine to thymine by thymidine phosphorylase liberated from lysed red cells (Bushby, 1973).

$E$. coli were isolated from patient D.F. (case 1) before treatment, immediately after co-trimoxazole therapy, and during convalescence. Of these, only the strain isolated immediately after treatment was thymidine-dependent. All three strains, however, were found to belong to the same serotype $(06 \cdot \mathrm{K}$ ? $\mathrm{H} 31$ ) and thymidine dependence was probably acquired and subsequently lost by mutations from the original parent strain. The $E$. coli which was isolated during the patient's convalescence was ampicillin resistant but apart from this the antibiotic sensitivity pattern was the same as the original strain.
Growth of thymidine-dependent strains varied on different batches of blood agar, ranging from scanty minute colonies to a luxuriant growth. The amount of growth appeared to be related to the thymidine content of the medium, which would in turn depend on the amount of thymidine present in the medium when prepared, and the degree to which it was subsequently neutralized by enzyme released from lysed blood cells.

Growth on different media was in accord with this explanation (table II). Nutrient agar and fresh Oxoid no. 2 blood agar clearly contained sufficient thymidine to support normal growth. Lysis of blood cells reduced the amount of growth; no growth occurred on lysed blood agar, but on chocolate agar, in which released enzyme would have been destroyed by heat, growth was normal. Growth was greatly reduced on an Oxoid no. 2 blood agar plate which was showing visible lysis, and even on four-day-old blood plates which were not visibly lysed, there was less growth than on fresh blood agar plates. It was also found that the thymidine content of the agar base used to make blood agar may be important. Thymidine-dependent mutants grew better on Oxoid no. 2 blood agar than on Columbia blood agar even though both were prepared with the same batch of horse blood at the same concentration.

\begin{tabular}{|c|c|c|c|c|}
\hline \multirow[t]{3}{*}{ Medium } & \multicolumn{3}{|c|}{ Growth of Thymidine-dependent E. coli } & \multirow{3}{*}{$\begin{array}{l}\text { Growth of Sensitive E. coli in the } \\
\text { Presence of Trimethoprim }\end{array}$} \\
\hline & \multicolumn{3}{|l|}{ Strain } & \\
\hline & D.F. & $T . T$. & E.H. & \\
\hline $\begin{array}{l}\text { Nutrient agar no. } 2 \\
\text { Chocolate agar } \\
\begin{array}{l}\text { Oxoid no. } 2 \text { blood agar Fresh } \\
\text { Four days old } \\
\text { Slightly lysed }\end{array} \\
\begin{array}{l}\text { Columbia blood agar Fresh } \\
\text { Lysed blood agar }\end{array}\end{array}$ & $\begin{array}{l}+++^{2} \\
+++ \\
+++ \\
++ \\
\mathbf{N G}^{3} \\
+ \\
\pm \\
\mathbf{N G}\end{array}$ & $\begin{array}{l}+++ \\
+++ \\
+++ \\
++ \\
+ \\
+ \\
\pm \\
\text { NG }\end{array}$ & $\begin{array}{l}+++ \\
+++ \\
+++ \\
++ \\
\text { NG } \\
+ \\
\pm \\
\text { NG }\end{array}$ & $\begin{array}{l}+++ \\
+++ \\
+++ \\
+ \\
\text { NG } \\
++ \\
+ \\
\text { NG }\end{array}$ \\
\hline
\end{tabular}

Table II Effects of different media on the growth of thymidine-dependent mutants of E. coli and on the growth of a sensitive E. coli in the presence of trimethoprim

${ }^{1}$ Growth of a trimethoprim-sensitive, non-thymidine-dependent $E$. coli in the immediate vicinity of a disc impregnated with $1 \cdot 25 \mu g$ trimethoprim. +++ normal size to \pm barely visible NG $=$ no growth 
Koch and Burchall (1971) have shown that the inhibitory activity of trimethoprim against a sensitive strain of $E$. coli varied inversely with the thymidine content of the medium. The growth of a sensitive $E$. coli in the presence of trimethoprim is therefore a measure of the thymidine content of the medium, and table II shows that such growth closely followed the pattern shown by thymidine-dependent mutants. This provides further evidence therefore that the extent of growth of these mutants was a reflection of the thymidine content of the medium.

\section{Discussion}

The acquisition of thymidine dependence by enterobacteria has been reported after about two weeks' co-trimoxazole treatment but, more commonly, it has been associated with prolonged courses of cotrimoxazole in patients with chronic urinary infection (Okubadejo and Maskell, 1973; Barker et al, 1972). Our patient D.F. (case 1) is interesting in that we witnessed the very rapid emergence of thymidinedependent mutants after only a few days of cotrimoxazole treatment. Her original $E$. coli, from which the mutant was apparently derived, was sulphonamide resistant, and using the disc technique no obvious synergy between trimethoprim and sulphonamide was found. Pinney and Smith (1973) showed that the mutation rate to double trimethoprim, sulphonamide resistance, and thymine dependence was of the order $2 \times 10^{-7}$ if the organisms harboured an $\mathbf{R}$ factor conferring sulphonamide resistance. They estimated that, in the absence of the $\mathbf{R}$ factor, the mutation rate to joint sulphonamide trimethoprim resistance would be of the order of $10^{-14}$. In retrospect it would have been wiser to have abandoned co-trimoxazole when it was found that the $E$. coli was sulphonamide resistant.

The ability to detect thymidine-dependent bacteria clearly depends on the availability of sufficient thymidine to support their growth. Koch and Burchall (1971) have shown that there is considerable variation in the amount of thymidine present in commercially available media and our experiments support this view. Moreover, the thymidine content of media may be reduced by lysed blood, and the gradual lysis which takes place in blood agar medium may significantly affect the growth of thymidinedependent organisms. It is important in clinical bacteriology therefore to remember that thymidinedependent bacteria may grow very poorly or not at all on blood agar.

We are grateful to $\mathrm{Dr} R$. Bolton for permission to publish details of patient D.F. (case 1) and to Dr B. Rowe of the Salmonella and Shigella Reference Laboratory, Colindale, for serotyping the $E$. coli strains.

\section{References}

Barker, J., Healing, D., and Hutchinson, J. G. P. (1972). Characteristics of some co-trimoxazole resistant Enterobacteriaceae from infected patients. J. clin. Path., 25, 1086-1088.

Bushby, S. R. (1973). Sensitivity testing with trimethoprim/sulphamethoxazole. Med. J. Aust., 1, Special Suppl. (June), 10-18.

Cowan, S. T., and Steel, K. J. (1966). Manual for the Identification of Medical Bacteria, Cambridge. Cambridge University Press.

Koch, A. E., and Burchall, J. J. (1971). Reversal of the antimicrobiol activity of trimethoprim by thymidine in commercially prepared media. Appl. Microbiol., 22, 812-817.

Okubadejo, O. A., and Maskell, R. M. (1973). Thymine-requiring mutants of Proteus mirabilis selected by co-trimoxazole in vivo. J. gen. Microbiol., 77, 533-535.

Pinney, R. J., and Smith, J. T. (1973). Joint trimethoprim and sulphamethoxazole resistance in bacteria infected with $R$ factors. J. med. Microbiol., 6, 13-19. 